\title{
Calculation of Microwave Land Surface Emissivity From Satellite Observations: Validity of the Specular Approximation Over Snow-Free Surfaces?
}

\author{
Fatima Karbou and Catherine Prigent
}

\begin{abstract}
To determine land surface emissivity from satellite microwave measurements, the surface is usually assumed to be specular. Questions about the validity of this approximation to estimate emissivity from nadir viewing radiometers were raised. This work aims to examine the validity of the specular assumption by evaluating errors induced when deriving emissivities from near-nadir measurements over snow-free areas. Brightness temperature simulations near nadir above both a specular and a Lambertian surface are compared. Errors on the retrieved emissivity introduced by the specular assumption are also quantified. The results show that the impact of the specular assumption when the surface is Lambertian is limited: less than $1 \%$ error in most atmospheric situations over natural snow-free surfaces.
\end{abstract}

Index Terms-Lambertian surface, microwave land emissivity, specular surface.

\section{INTRODUCTION}

$\mathbf{T}$ O ESTIMATE land surface microwave emissivities from satellite observations, the specular approximation is generally adopted. It is a practical solution that can be used without a priori information on the surface in the case of global emissivity calculations. This approximation has been used by several investigators to calculate emissivities from the Special Sensor Microwave/Imager (SSM/I) conical scanner with incident angle around $53^{\circ}$ (e.g., [1], [7], and [9]) and also from Advanced Microwave Sounding Unit (AMSU) cross-track scanners [3], [8].

Questions have recently been raised about the applicability of this approximation to close-to-nadir observations. Calculations and an experiment by Matzler [4] tend to show that the contribution of the Lambertian component can be significant in some very specific conditions, for instance over a metallic surface in the laboratory. He then suggests to characterize the surface with a specularity parameter that would account for the partitioning between the specular and the Lambertian contributions to the emissivity. However, no realistic practical solution is given to

Manuscript received January 10, 2005; revised February 10, 2005. F. Karbou acknowledges financial support from the Centre National d'Etudes Spatiales (CNES).

F. Karbou is with the Centre National de Recherches Météorologiques (CNRM), GAME, Centre National de la Recherche Scientifique (CNRS), 31057 Toulouse, France (e-mail: fatima.karbou @ cnrm.meteo.fr).

C. Prigent is with the Centre National de la Recherche Scientifique (CNRS), Observatoire de Paris, 75014 Paris, France.

Digital Object Identifier 10.1109/LGRS.2005.847932 derive such a parameter on a global basis. The purpose of this letter is to objectively quantify the error associated to the specular approximation for emissivity calculations from real satellite observations in snow-free regions. First the specular and the Lambertian approximations will be briefly recalled. Then the two approximations will be used to estimate the emissivity from AMSU observations for one month over a large portion of the earth. The differences related to the two approximations will be evaluated both in terms of brightness temperatures and retrieved emissivities.

\section{SPECUlAR VERSUS THE LAMBERTIAN APPROXIMATIONS}

Over a flat lossy surface, the integrated radiative transfer equation in the Rayleigh-Jeans approximation, for a nonscattering plane-parallel atmosphere, can be expressed in terms of brightness temperature for a given polarization state $p$

$$
\begin{aligned}
T b_{P}(\theta) & =T_{\mathrm{SURF}} \times \varepsilon_{P}(\theta) \times e^{-\frac{\tau(0, H)}{\cos (\theta)}}+ \\
\cdot & T_{\mathrm{ATM}}^{\downarrow}(\theta) \times\left(1-\varepsilon_{P}(\theta)\right) \times e^{-\frac{\tau(0, H)}{\cos (\theta)}}+T_{\mathrm{ATM}}^{\uparrow}(\theta)
\end{aligned}
$$

with

$$
T_{\mathrm{ATM}}^{\uparrow}(\theta)=\int_{0}^{H} T(z) \frac{\alpha(z)}{\cos (\theta)} e^{\frac{-\tau(z, H)}{\cos (\theta)}} d z .
$$

$T b_{p}(\theta)$ is the brightness temperature measured by the satellite for polarization state $p$ and incidence angle $\theta ; T_{\text {SURF }}$ is the surface "skin" temperature; $\varepsilon_{p}(\theta)$ is the surface emissivity for polarization state $p$ and incidence angle $\theta ; \alpha(z)$ is the atmospheric absorption by gases at altitude $z ; T(z)$ is the atmospheric temperature at altitude $z ; \tau(z 0, z 1)=\int_{z 0}^{z 1} \alpha(z) d z$ is the atmospheric extinction from $z 0$ to $z 1$; and $H$ is the orbiter height.

In the case of a specular reflection

$$
T_{\mathrm{ATM}}^{\downarrow}(\theta)=\int_{H}^{0} T(z) \frac{\alpha(z)}{\cos (\theta)} e^{-\frac{\tau(z, 0)}{\cos (\theta)}} d z .
$$

It means that the downwelling radiation reflected by the surface only comes from the specular angle $\theta$. It is strictly valid for very smooth surfaces only. A specular reflection off-nadir polarizes the signal. 


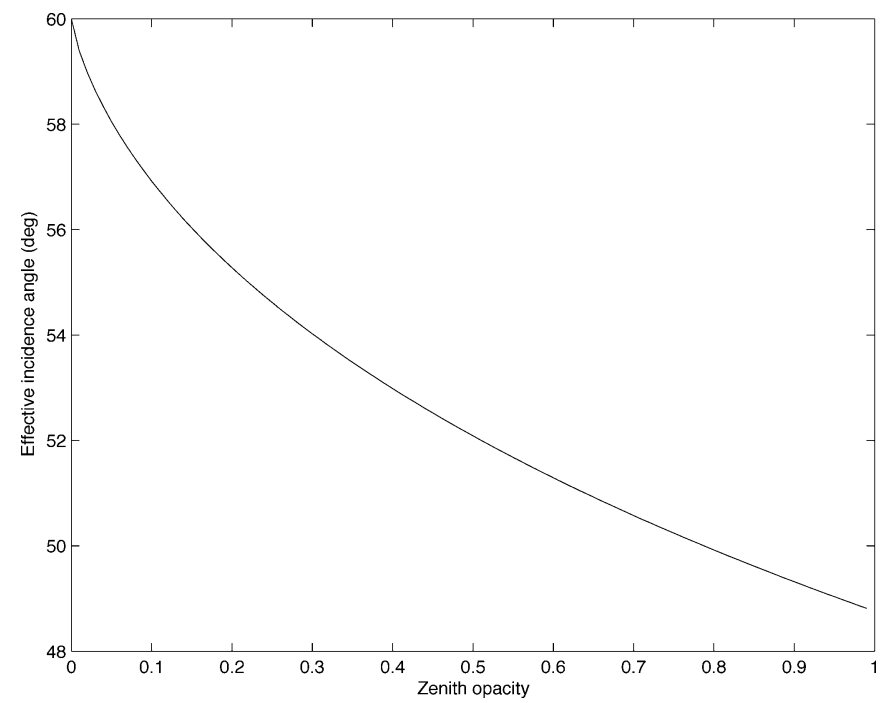

Fig. 1. Effective incidence angle as a function of the zenith opacity.

On the other extreme, for very rough surfaces that are characterized by Lambertian reflection, the downwelling radiations are isotropically reflected by the surface and no polarization is generated

$$
\begin{aligned}
T_{\mathrm{ATM}}^{\downarrow}(\theta)=\int_{0}^{\pi / 2} 2 \cos (\theta) & \sin (\theta) \\
& \cdot\left(\int_{H}^{0} T(z) \frac{\alpha(z)}{\cos (\theta)} e^{-\frac{\tau(z, 0)}{\cos (\theta)}} d z\right) d \theta .
\end{aligned}
$$

Matzler [5] and Ingold et al. [2] derived an effective incidence angle $\theta_{\text {eff }}$ that would correspond to the downwelling radiation and showed that this angle is given by

$$
\theta_{\mathrm{eff}}=\arccos \left(\frac{-\tau}{\ln \left(2 \times E_{3}(\tau)\right)}\right)
$$

with $E_{3}$ the exponential integral of order 3 .

The downwelling radiation in the Lambertian approximation is easily calculated from (2) substituting $\theta$ by $\theta_{\text {eff. }}$. Calculations of $\theta_{\text {eff }}$ from (4) is presented in Fig. 1 as a function of the zenith opacity. For window channels, the zenith opacity is close to 0.1 and in this case, $\theta_{\text {eff }}$ is close to $55^{\circ}$. This justifies the specular reflection whatever the surface type in the case of conical scanners with incidence angle close to $53^{\circ}$.

Using a limited set of Tbs and emissivity simulations, Matzler [4] concludes that the use of the specular assumption is questionable when using nadir viewing satellite data. The author compares nadir Tbs above a specular surface and above a Lambertian surface assuming an initial emissivity of 0.8 and a standard atmosphere. Then, emissivities are derived from the simulated Tbs with a specular surface reflection in order to quantify errors introduced by the surface reflection type. The results are reproduced on Fig. 2(a) and (b) for Tbs and emissivity comparisons respectively. With an initial emissivity of 0.8 and at 0.1 zenith opacity, the Tbs difference above Lambertian and specular surfaces could reach $3.7 \mathrm{~K}$ inducing an emissivity overestimation of $1.5 \%$ if the surface is Lambertian. The Tbs difference
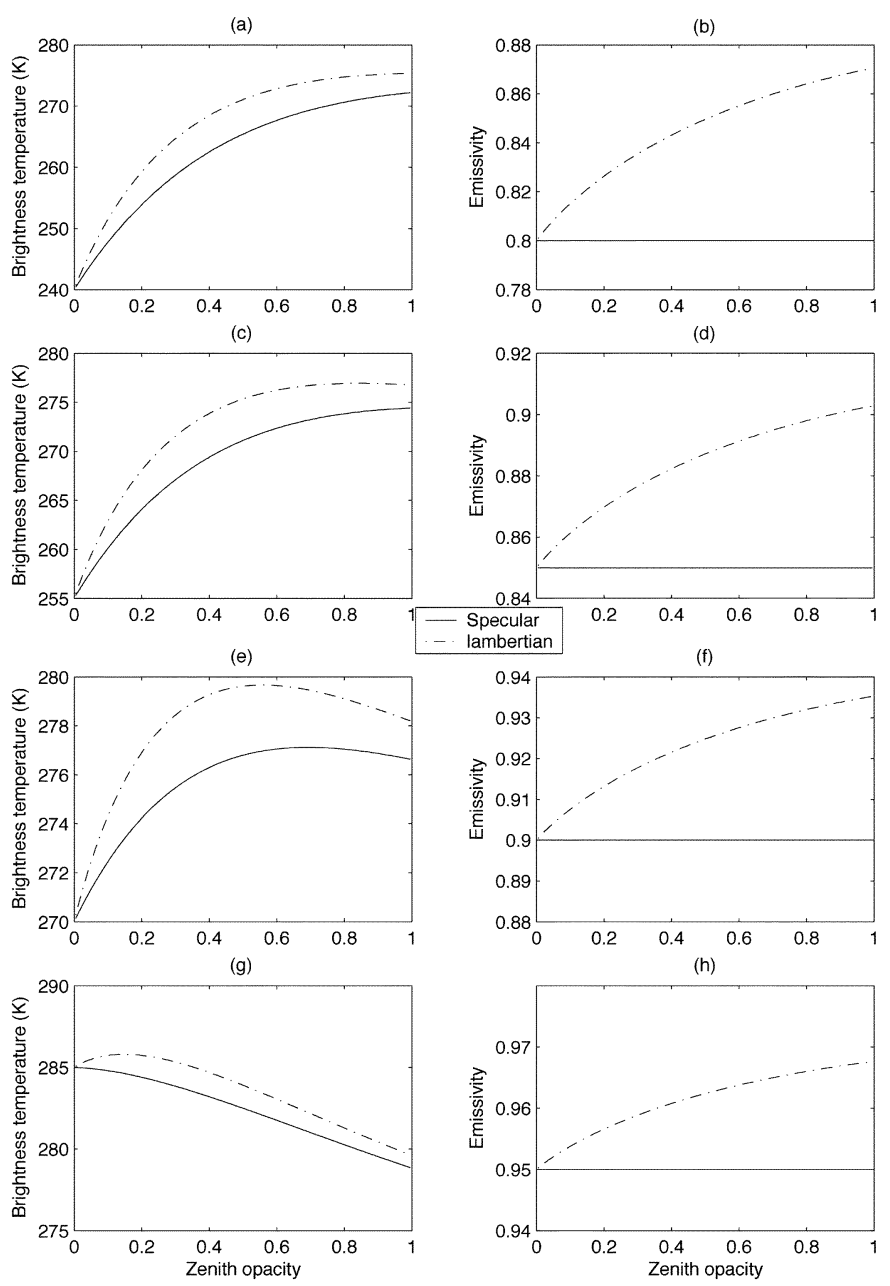

Fig. 2. (a) Tbs at nadir as a function of the zenith opacity over a Lambertian surface (dashed-dotted line) and a specular surface (solid line) with an initial emissivity of 0.8 and a standard atmosphere. (b) The derived emissivities from the previous specular Tbs (solid line) and Lambertian ones (dashed-dotted line) at nadir assuming a specular surface. (c), (e), and (g) are same as (a) but with an initial emissivity of $0.85,0.9$, and 0.95 , respectively. (d), (f), and (h) are same as (b) but with an initial emissivity of $0.85,0.9$, and 0.95 , respectively.

first increases with increasing zenith opacity as the downwelling $\mathrm{Tb}$ in (1) increases. Then it decreases due to the fact that the surface contribution decreases with atmospheric opacity. The differences in emissivity keeps increasing with zenith opacity: note that when estimating emissivities from satellite observations, situations with large zenith opacity have to be avoided because the surface contribution to the Tbs is then very limited and the emissivity calculation would have larger errors.

The snow- and water-free surfaces are usually associated to rather high emissivity (usually more than 0.95 ). Fig. 3 shows in (a) the monthly mean emissivity map at $19 \mathrm{GHz}((E v+E h) / 2)$ using data from SSM/I during February 1993 (from [7]) and (b) the mean emissivity difference map between vertical and horizontal polarizations for the same channel. As expected, the emissivity is higher over vegetated areas (almost 0.98 ) than over bare soils (close to 0.9). Bare soil are associated with lower mean emissivities but also have higher emissivity polarization differences. These maps illustrate that for most natural surfaces the emissivity is rather high (and as a consequence the weight of the $T_{\mathrm{ATM}}^{\downarrow}$ is limited) and that lower emissivities are usually 
(a)

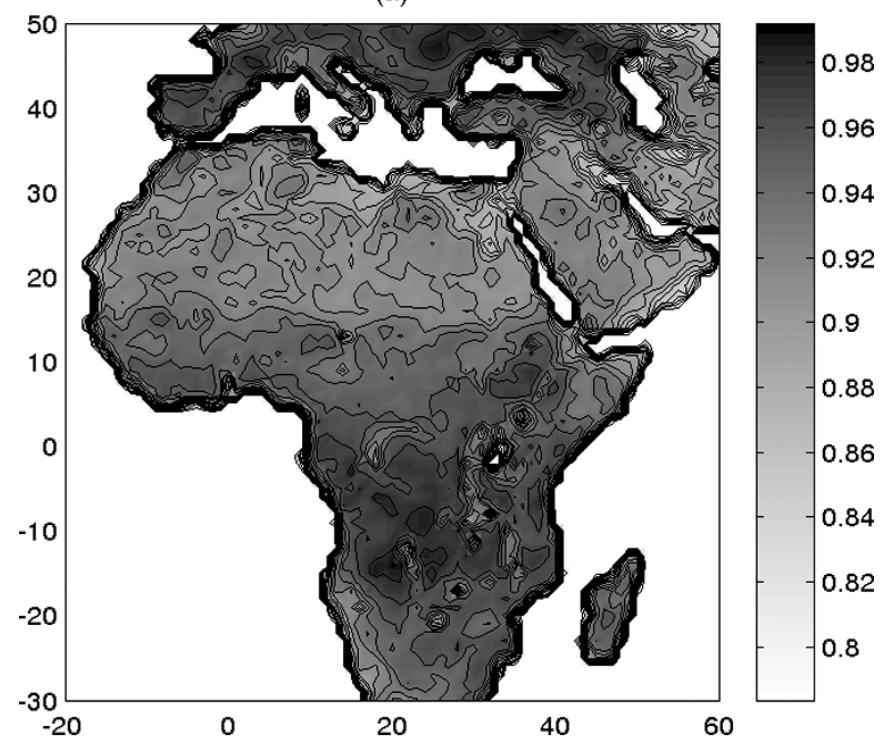

(b)

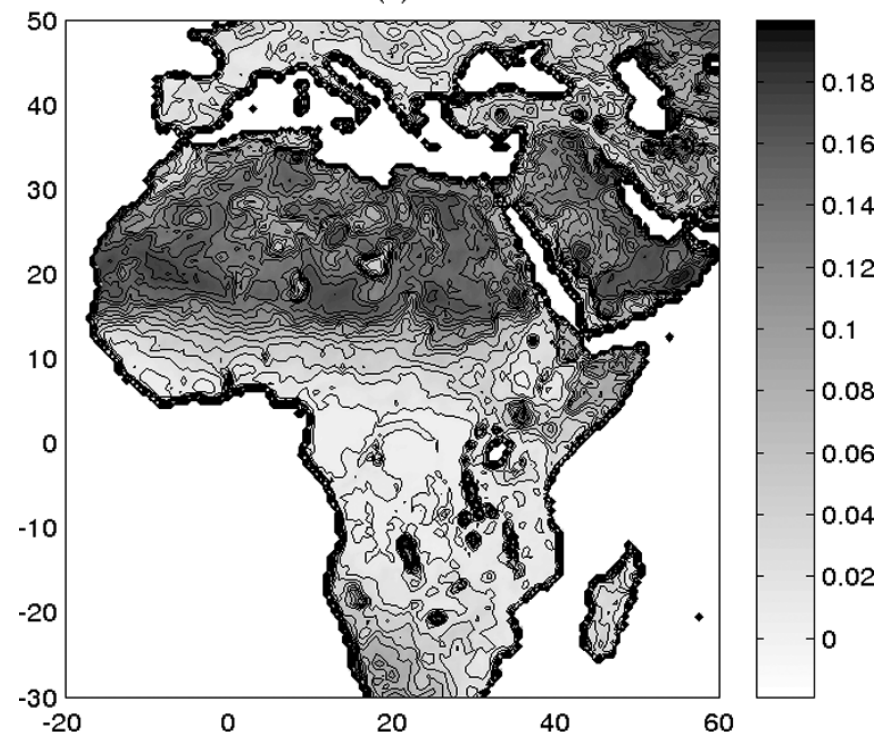

Fig. 3. (a) Monthly mean emissivity map at $19 \mathrm{GHz}((E v+E h) / 2))$ using data from SSM/I during February 1993 and (b) the mean emissivity difference map between vertical and horizontal polarizations for the same channel.

polarized (i.e., have a significant specular contribution, Lambertian surfaces being unpolarized).

Simulations similar to those carried out by Matzler [4] [shown in Fig. 2(a) and (b)] are performed using the same atmospheric situation but with an initial emissivity varying from $0.85-0.95$. The results are presented in subplots [2(c)-(h)]. With identical surface and atmospheric situations, the differences between Tbs above specular and Lambertian surfaces are less important with the new emissivity values than with an emissivity of 0.8 . At zenith opacity of 0.1 , the Tbs difference is close to $2.8,1.9$, and $0.9 \mathrm{~K}$ using an initial emissivity of $0.85,0.9$, and 0.95 , respectively. Consequently, the emissivity would be overestimated by about $1.1 \%, 0.7 \%$, and $0.3 \%$ if the surface was purely Lambertian.

In the next section, emissivity retrievals over both surface types are performed using data near nadir from AMSU-A instrument on board NOAA-15 satellite in order to investigate the

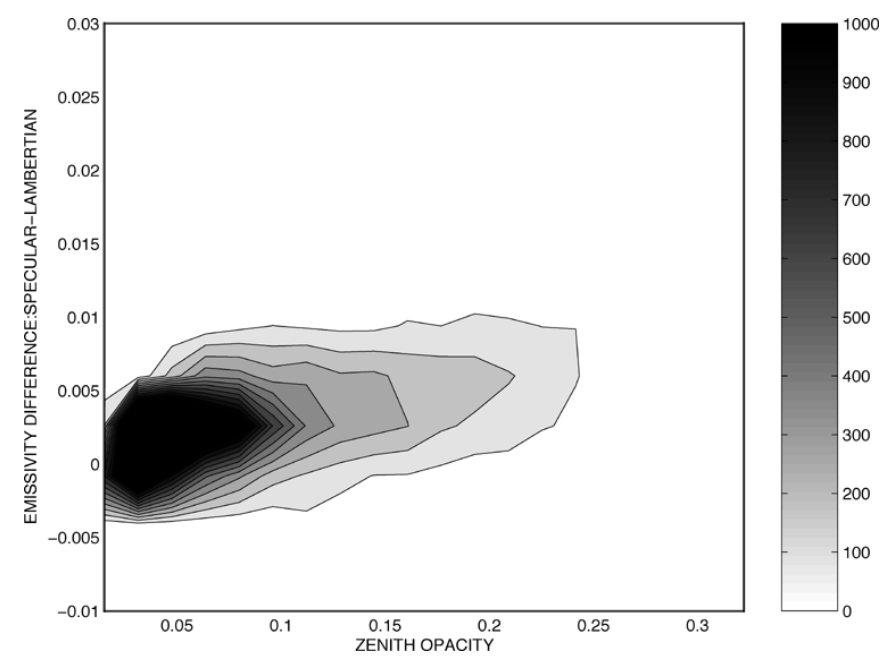

Fig. 4. Density contours of the emissivity differences between the specular and the Lambertian cases as a function of the zenith opacity, using data near nadir from AMSU-A instrument at $23 \mathrm{GHz}$ and during February 2000.

validity of the specular approximation in most atmospheric and surface conditions.

\section{APPliCATION OF THE TwO APPROXIMATIONS TO REAL SATELLITE OBSERVATIONS AND COMPARISON}

Microwave land surface emissivities are calculated for close-to-nadir conditions from AMSU-A NOAA-15 observations, using ECMWF and ISCCP ancillary information to accurately determine the atmospheric and surface contributions. The ISCCP dataset provides information about clouds and skin temperature whereas European Centre for Medium-Range Weather Forecasts temperature-humidity profiles are used to input an up-to-date radiative transfer model [6] to estimate the cloud- and rain- free atmospheric contribution to the measured radiances. The emissivity can then be calculated as follows:

$$
\varepsilon_{P}(\theta)=\frac{T b_{P}(\theta)-T_{\mathrm{ATM}}^{\uparrow}(\theta)-\left(T_{\mathrm{ATM}}^{\downarrow}(\theta) \times e^{-\frac{\tau(0, H)}{\cos (\theta)}}\right)}{\left(T_{\mathrm{SURF}}-T_{\mathrm{ATM}}^{\downarrow}(\theta)\right) \times e^{-\frac{\tau(0, H)}{\cos (\theta)}}} .
$$

The surface is considered first as specular then as Lambertian to derive two emissivity datasets. The method described in Section II is used to estimate emissivity above a Lambertian surface. The calculations are performed for a large geographic area using data from February 2000 at $23.8 \mathrm{GHz}$. Fig. 4 presents density contours of the emissivity differences between the specular and the Lambertian cases as a function of the zenith opacity. It shows that for most situations, differences associated to the specular and Lambertian approximations are very limited, well within $1 \%$. Fig. 5 completes this analysis by showing mean emissivity difference (in percent) map at $23.8 \mathrm{GHz}$ between the specular and the Lambertian cases. At $89 \mathrm{GHz}$, the emissivity differences are larger than those obtained at $23.8 \mathrm{GHz}$ but still well within $1.5 \%$ over most areas. Larger values are associated with high zenith opacity. However, it should be noted that observations with zenith opacity greater than 0.4 should not be considered for emissivity calculations because the surface is hardly visible in such conditions. When such cases are removed from 


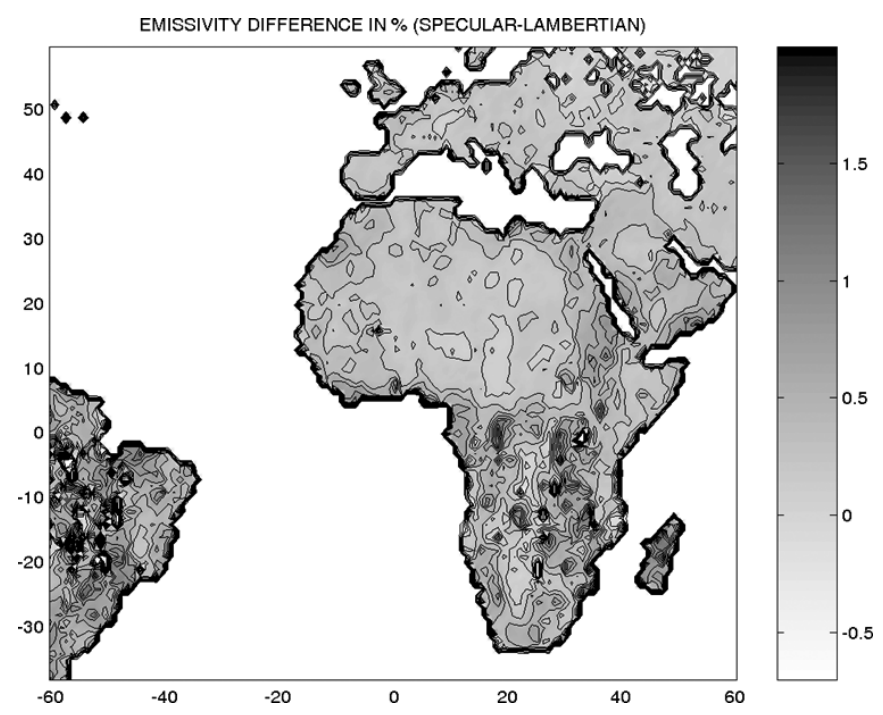

Fig. 5. Mean emissivity difference (in percent) map between the specular and the Lambertian cases using data near nadir from AMSU-A instrument at $23 \mathrm{GHz}$ and during February 2000.

our database, the general statistics (mean/std) over the entire geographic area are: $0.007 / 0.007$. It is clear that emissivity estimations from satellite observations have to be averaged over a certain period of time, to reduce emissivity errors especially at high frequencies. The emissivity averaging is required not only for surface approximation induced errors but also for errors induced by input parameters, such as the skin temperature and the humidity profiles. In a previous work, we studied the emissivity sensitivity to errors in the input parameters and we showed that the impact of some of them is not negligible at all frequencies [3].

\section{CONCLUSION}

The specular approximation is not strictly valid for all surface types and observation conditions. However, this note confirms that the error related to the specular approximation is limited, well within $1 \%$ even for close-to-nadir observations in the case of natural snow-free surfaces as measured from satellites. Large differences between the two approximations have been calculated or observed for very specific cases as reported in [4], but they are not representative of natural snow-free surfaces over land. Introducing a specularity parameter could eventually reduce the already limited noise associated to the specular approximation but anyhow, no method exists yet to derive such a parameter on a global basis.

\section{ACKNOWLEDGMENT}

The authors are very grateful to two anonymous reviewers as well as to the associate editor for detailed and helpful comments about the manuscript.

\section{REFERENCES}

[1] A. S. Jones and T. H. Vonder Haar, "Retrieval of microwave surface emittance over land using coincident microwave and infrared satellite measurements," J. Geophys. Res., vol. 102, no. D12, pp. 13 609-13,626, 1997.

[2] T. Ingold, R. Peter, and N. Kampfer, "Weighted mean tropospheric temperature and transmittance determination at millimeter-wave frequencies for ground-based applications," Radio Sci., vol. 33, no. 4, pp. 905-918, 1998.

[3] F. Karbou, C. Prigent, L. Eymard, and J. Pardo, "Microwave land emissivity calculations using AMSU measurements," IEEE Trans. Geosci. Remote Sens., vol. 43, no. 5, pp. 948-959, May 2005.

[4] C. Matzler, "On the determination of surface emissivity from satellite observations," IEEE Geosci. Remote Sensing Lett., no. 2, pp. 160-163, Apr. 2005.

[5] - "Applications of the interaction of microwaves with the natural snow cover," Remote Sens. Rev., vol. 2, no. 12, pp. 259-392, 1987.

[6] J. R. Pardo, J. Cernicharo, and E. Serabyn, "Atmospheric Transmission at Microwave (ATM): An improved model for millimeter/submillimeter applications," IEEE Trans. Antennas Propagat., vol. 49, no. 12, pp. 1683-1694, Dec. 2001.

[7] C. Prigent, W. B. Rossow, and E. Matthews, "Microwave land surface emissivities estimated from SSM/I observations," J. Geophys. Res., vol. 102, pp. $21867-21890,1997$.

[8] C. Prigent, F. Chevallier, F. Karbou, P. Bauer, and G. Kelly, "AMSU-A land surface emissivity estimation for numerical weather prediction assimilation schemes," J. Appl. Meteorol., 2005, to be published.

[9] F. Weng, B. Yan, and N. C. Grody, "A microwave land emissivity model," J. Geophys. Res., vol. 106, pp. 20115-20123, 2001. 2001.21867-21890. 